\title{
O PROCESSO PSICOTERAPÊUTICO PSICODINÂMICO DE UM MENINO COM SINTOMAS INTERNALIZANTES: UMA ANÁLISE COM BASE NO CHILD PSYCHOTHERAPY Q-SET
}

\author{
THE PSYCHODYNAMIC PSYCHOTHERAPEUTIC PROCESS OF A BOY WITH INTERNALIZING \\ SYMPTOMS: AN ANALYSIS BASED ON THE CHILD PSYCHOTHERAPY Q-SET \\ EL PROCESO PSICOTERAPÉUTICO PSICODINÁMICO DE UN NIÑO CON SÍNTOMAS DE \\ INTERNALIZACIÓN: UN ANÁLISIS CON BASE EN EL CHILD PSYCHOTHERAPY Q-SET
}

\author{
Maiara Castro de Freitas* \\ Cibele Carvalho \\ Vera Regina Röhnelt Ramires ${ }^{* * *}$
}

\begin{abstract}
RESUMO
Problemas internalizantes são frequentes em crianças, mas os ingredientes ativos que tornam uma psicoterapia efetiva são ainda pobremente compreendidos. O objetivo deste estudo foi analisar o processo terapêutico psicodinâmico de um menino em idade escolar que apresentava sintomas internalizantes, cujo tratamento foi interrompido após 22 meses. Os participantes foram o paciente e sua terapeuta. O Child Psychotherapy Q-Set foi utilizado para analisar o processo terapêutico. Os resultados mostraram as características da relação terapeuta-paciente e suas transformações. Fatores como conflitos familiares, aliança terapêutica com os pais, limitação da capacidade de mentalização do menino, contratransferência, habilidades e ajustamento da terapeuta ao paciente tiveram impacto no processo terapêutico, contribuindo para o desfecho observado. Para elucidar questóes em aberto acerca da efetividade e mecanismos de mudança da psicoterapia psicodinâmica, e para estabelecimento de uma base de evidências consistente, é imprescindível que se conheça, de fato, o que ocorre e como ocorre no setting clínico.
\end{abstract}

Palavras-chave: Psicoterapia de crianças. Processo psicoterapêutico. Transtornos internalizantes. Estudo de caso único.

\footnotetext{
Texto recebido em 13 de março de 2017 e aprovado para publicação em 4 de julho de 2018.

Este estudo foi financiado pela CAPES, com bolsa de estudos pelo Programa Prosup, e pelo Conselho Nacional de Desenvolvimento Científico e Tecnológico do Brasil (CNPq), Chamada MCTI/CNPq/MEC/CAPES no 22/2014 Ciências Humanas, Sociais e Sociais Aplicadas e Chamada Produtividade em Pesquisa - PQ 2014, processos 471358/2014-2 e 311235/2014-0.

* Mestra em Psicologia Clínica pela Unisinos, psicóloga. Endereço: Rua Marechal Mallet, no 701 - Monsenhor Assis, Santiago-RS, Brasil. CEP: 97700-000. E-mail: freitas_may@hotmail.com

** Mestra e doutoranda em Psicologia Clínica pela Unisinos, psicóloga. E-mail: cibele.carvalho8@gmail.com

*** Doutora em Psicologia Clínica pela PUC de São Paulo, professora e pesquisadora no Programa de Pós-Graduação em Psicologia da Unisinos, psicóloga. E-mail: verareginaramires@gmail.com
} 


\begin{abstract}
Internalizing problems are frequent in children, but the active ingredients that make a psychotherapy effective are still poorly understood. This study aimed to analyze the psychodynamic therapeutic process of a school-age boy who presented internalizing symptoms, whose treatment was interrupted after 22 months. Participants were the patient and the therapist. The Child Psychotherapy Q-Set was used to analyze the therapeutic process. The results showed the characteristics of the therapist-patient relationship and its transformations. Factors such as family conflicts, therapeutic alliance with the parents, limitation to the ability to mentor the boy, countertransference, skills, and adjustment of the therapist to the patient impacted the therapeutic process, contributing to the observed outcome. In order to clarify the questions about the effectiveness and mechanisms of change in psychodynamic psychotherapy, and for the establishment of a consistent base of evidence, it is essential to know, in fact, what happens and how it happens in the clinical setting.
\end{abstract}

Keywords: Psychotherapy of children. Psychotherapeutic process. Internalizing disorders. Single case study.

\title{
RESUMEN
}

Problemas de internalización son frecuentes en niños, pero los ingredientes activos que hacen que una psicoterapia sea efectiva todavía son pobremente comprendidos. El objetivo de este estudio fue analizar el proceso terapéutico psicodinámico de un niño en edad escolar que presentaba síntomas de internalización y cuyo tratamiento fue interrumpido después de 22 meses. Los participantes fueron el paciente y su terapeuta. El Child Psychotherapy Q-Set fue utilizado para analizar el proceso terapéutico. Los resultados mostraron las características de la relación terapeuta-paciente y sus transformaciones. Factores como conflictos familiares, alianza terapéutica con los padres, limitación de la capacidad de mentalización del niño, contratransferencia, habilidades y ajustamiento de la terapeuta al paciente tuvieron impacto en el proceso terapéutico, contribuyendo para el desenlace observado. Para elucidar cuestiones en abierto acerca de la efectividad y de los mecanismos de cambio de la psicoterapia psicodinámica, y para el establecimiento de una base de evidencias consistente, es imprescindible que se sepa, de hecho, qué ocurre y cómo ocurre el setting clínico.

Palabras clave: Psicoterapia infantil. Proceso psicoterapéutico. Trastornos de internalización. Estudio de caso único. 


\section{INTRODUÇÃO}

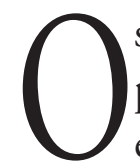

$s$ problemas psicológicos apresentados pela população infantil podem gerar prejuízos importantes para seu desenvolvimento, funcionamento familiar e contexto social. Algumas dificuldades emocionais e de comportamento que se manifestam na infância podem predizer psicopatologias na idade adulta (Mash, \& Hunsley, 2010). Além disso, se não tratados adequadamente, esses transtornos tendem a persistir e aumentam o risco para o desenvolvimento de outras desordens (Göttken, White, Klein, \& Klitzing, 2014). Apesar disso, poucas crianças e adolescentes obtêm tratamento ou suporte terapêutico adequado (Fonagy, Target, Cottrell, Phillips, \& Kurtz, 2005).

A literatura tem reunido tais dificuldades emocionais e comportamentais infantis em dois grupos: problemas internalizantes e externalizantes (Achenbach, 1991). Os problemas internalizantes são vivenciados internamente pela criança e contemplam depressão, ansiedade, desordens obsessivo-compulsivas, queixas somáticas, retraimento, sentimento de inferioridade, medo e tristeza. Por outro lado, as desordens disruptivas, ou problemas externalizantes, referem-se à conduta desafiadora excessiva e transtornos de conduta-agressividade (a pessoas e animais), favorecendo os conflitos com o ambiente (American Psychiatric Association, 2014; Wilkinson, 2009).

Entretanto, apesar de seu impacto prejudicial ao desenvolvimento socioemocional, os problemas internalizantes têm sido subidentificados $\mathrm{e}$ subtratados (Flett, \& Hewitt, 2013). Entre os problemas emocionais na idade escolar, o transtorno de ansiedade (TA) é considerado o mais comum, abrangendo de $4 \%$ a $25 \%$ de crianças (Boyd, Kostanski, Gullone, Ollendick, \& Shek, 2000). No entanto, os sintomas de TA são difíceis de identificar e tratar, pois não costumam afetar diretamente amigos, familiares e outras pessoas do convívio da criança (Bayer et al., 2011).

Na maioria dos casos, os TA estão associados à depressão (Fonagy et al., 2005), desordem cada vez mais frequente e precoce em crianças (Goldmann, 2012; Merry et al., 2012). Na prática, selecionar um tratamento apropriado a esses problemas tem sido difícil. A psicoterapia parece ser uma escolha importante, no entanto os estudos ainda são muito restritos.

A psicoterapia individual deve oferecer oportunidades à criança para articular pensamentos, sentimentos, lembranças e desejos, para fazer conexões entre seus sentimentos e conflitos inconscientes (Fonagy et al., 2005). Nesse sentido, a psicoterapia psicodinâmica (PP) mostra-se indicada, visto que pretende auxiliar o indivíduo a obter um aumento do conhecimento de seu funcionamento, o que 
resulta no uso de defesas mais maduras para lidar com conflitos psíquicos, na melhora do padrão das relações objetais e em mudanças sintomáticas significativas (Eizirik, \& Hauck, 2008).

Muitos clínicos e pesquisadores, como visto em revisões de literatura e ensaios clínicos, sugerem o tratamento combinado, com associação da PP e alguma medicação direcionada ao transtorno (Bernstein, \& Shaw, 1997; Birmaher, \& Brent, 2007; Muratori, Picchi, Bruni, Patarnello, \& Romagnoli, 2003; Wehry, Beesdo-Baum, Hennelly, Connolly, \& Strawn, 2015). Alguns estudos, na última década, sobre a efetividade da PP com crianças e adolescentes, demonstraram os benefícios desse tratamento (Deakin, \& Nunes, 2008; Midgley, \& Kennedy, 2011; Palmer et al., 2013). A PP de crianças tem sido indicada no tratamento dos transtornos internalizantes (Midgley, \& Kennedy, 2011; Palmer, Nascimento, \& Fonagy, 2013). No entanto, as evidências são limitadas, pois poucos estudos foram realizados nessa área (Fonagy et al., 2005).

Diante do importante papel desempenhado pela psicoterapia nos tratamentos de crianças com problemas internalizantes, e visto que a intervenção precoce pode reduzir a gravidade ou persistência do transtorno, destaca-se a importância de mais estudos na área, principalmente para se avaliar quais fatores, no processo terapêutico, contribuem para exercer um papel significativo na melhora da criança.

\subsection{ESTUDOS SOBRE O PROCESSO PSICOTERAPÊUTICO PSICANALÍTICO DE CRIANÇAS COM PROBLEMAS INTERNALIZANTES}

A literatura indica a necessidade de compreender-se mais sobre o processo psicoterápico, entender como e por que as mudanças ocorrem e quais aspectos do tratamento podem facilitar ou inibir mudanças terapêuticas (Palmer et al., 2013; Schneider, Midgley, \& Duncan, 2010). Diante dessa perspectiva, identifica-se que a pesquisa de processo de psicoterapia de crianças ampliou-se nos últimos anos. Schneider, Pruetzel-Thomas e Midgley (2009), por exemplo, utilizaram o Child Psychotherapy Q-Set (CPQ), instrumento que permite a descrição do processo terapêutico de crianças de 3 a 13 anos de idade, para estudar quatro psicoterapias de orientação psicanalítica e duas com abordagem da TCC. Os pacientes tinham entre 8 e 12 anos de idade e tinham sintomas de ansiedade e, ou, depressão. Foram avaliadas três sessões de cada um dos tratamentos. Os resultados mostraram que existem semelhanças nas características do processo terapêutico de crianças com o mesmo sintoma, mesmo quando atendidas por diferentes terapeutas. Contrariamente, o mesmo terapeuta com diferentes 
crianças que exibiam dificuldades distintas apresentou padrões de interação diferentes. Além disso, os autores constataram que as técnicas que caracterizam a PP foram consideravelmente ausentes na TCC e vice-versa.

Posteriormente, Schneider et al. (2010) avaliaram o processo terapêutico de uma criança com 11 anos de idade que apresentava, de acordo com o DSM-IV, transtorno depressivo maior, transtorno de ansiedade generalizada, transtorno do pânico e transtorno evitativo da infância. $O$ tratamento psicodinâmico durou três anos, com quatro sessões semanais. Para análise desse processo, utilizaramse de nove sessões (três do início, três do meio e três do fim). A menina foi descrita, no início da psicoterapia, como extremamente "fechada em si mesma", com baixa autoestima e dificuldades de concentração. No final do tratamento, foi relatado que ela se envolvia em atividades escolares, mantendo interesse vivo em seu mundo. Ainda apresentava atitude reservada, mas havia começado a socializar e fazer amigos. Além disso, sua relação com a mãe havia melhorado. Constatou-se que a integração de intervenções suportivas e interpretativas por parte da terapeuta auxiliaram na melhora sintomática da criança. As mudanças ocorreram, também, em razão dos processos interacionais da menina com a terapeuta, que se mostrou empática, modelou as emoções da criança, validando sua experiência, utilizando a interpretação para ajudá-la a compreender e elaborar pensamentos e sentimentos.

Schmidt (2016) investigou o processo terapêutico psicodinâmico de uma menina com 8 anos de idade, diagnosticada com transtorno de adaptação. A menina apresentava queixas somáticas sem causas orgânicas. Tinha crises de enxaqueca, de choro, dores de estômago e dizia não gostar da escola. Seu pai havia falecido repentinamente, e mudanças marcavam sua nova estrutura familiar e financeira. A terapeuta mostrou-se sensível e engajada, utilizando de diversas técnicas para auxiliar a menina na comunicação de seus pensamentos, sentimentos e na compreensão de seus significados. Ao longo de todo o tratamento, a menina comunicou-se com afeto bem modulado, respondendo com jogo mais elaborado às intervenções da terapeuta e mostrando-se aberta a examinar seus conflitos. No fim do tratamento, estava mais desinibida e segura, expressando-se de forma clara e fluente, alcançando novas compreensões e insights.

Recentemente, Carvalho, Godinho e Ramires (2016) analisaram o primeiro ano do processo terapêutico psicodinâmico de um menino com 7 anos de idade. O CPQ foi utilizado em duas sessões do início do tratamento, duas sessões após três meses, duas sessões após seis meses, duas sessões após nove meses e duas sessões após um ano. Buscou-se identificar as características do processo, ao longo do tempo. Os pais buscaram atendimento devido às dificuldades de relacionamento que o filho apresentava na escola e, também, por demonstrar alguns sinais de 
ansiedade. Os resultados indicaram que as atitudes da terapeuta, no começo da psicoterapia, envolveram um trabalho interpretativo e, mais tarde, de apoio e reflexão. $\mathrm{O}$ menino mostrou-se inicialmente resistente e com poucos recursos internos. No entanto, ao longo do tratamento, demonstrou maior abertura ao trabalho terapêutico, contemplando conteúdos importantes para seus conflitos.

Esses resultados vão ao encontro de outros estudos, quer sejam ensaios clínicos ou revisões de literatura sobre a PP de crianças com problemas internalizantes, e ratificam a efetividade desse tratamento (Bernstein, \& Shaw, 1997; Göttken et al., 2014; Milrod et al., 2013). As melhoras sintomáticas e psicossociais são evidenciadas ao longo de todo o tratamento, sendo que as crianças passaram a ter um funcionamento menos prejudicado após as intervençôes psicodinâmicas.

Nem sempre, contudo, os tratamentos são bem-sucedidos. Algumas vezes, eles podem ser interrompidos ou finalizar sem que os resultados almejados sejam atingidos. Estudos desenvolvidos em contexto brasileiro descreveram índices de interrupção na psicoterapia de crianças que variaram de $25 \%$ a $60 \%$ entre os casos pesquisados (Deakin, Gastaud, \& Nunes, 2012; Deakin, \& Nunes, 2008). Diante disso, a investigação do processo terapêutico pode contribuir para a compreensão das características da PP com crianças, identificando aspectos importantes do processo que promovam seu sucesso e elucidem seu fracasso, quando ocorrer. Assim, esta pesquisa foi delineada com o objetivo de analisar, por meio do $\mathrm{CPQ}$, o processo psicoterapêutico psicodinâmico de um menino que apresentava sintomas internalizantes, cujo tratamento foi interrompido após 22 meses. Buscou-se descrever as principais características da interação terapeutapaciente assim como aspectos relacionados à criança e ao terapeuta nas sessões, na tentativa de compreender a trajetória e o desfecho dessa psicoterapia.

\section{MÉTODO}

\subsection{DELINEAMENTO}

Foi realizado um estudo exploratório e descritivo, longitudinal, caracterizado como um estudo de processo e que adotou o método de estudo de caso sistemático (ECS) (Edwards, 2007).

\subsection{PARTICIPANTES}

Participaram deste estudo uma criança e sua terapeuta. O paciente, Antônio (pseudônimo) tinha 9 anos no início de sua psicoterapia, cursava o terceiro ano 
do ensino fundamental e residia com a mãe, o pai e o irmão mais novo. Os pais buscaram atendimento para o filho por estarem preocupados e não saberem lidar com seu comportamento agressivo. A mãe relatou que o filho era tímido, roía as unhas, não conseguia perder quando jogava com os amigos ou com a família e que ficava muito brabo quando isso acontecia. $\mathrm{O}$ irmão mais novo foi diagnosticado com "traços autistas" e, segundo os pais, isso vinha tomando muito o tempo deles. Também relataram que Antônio estava reclamando de falta de atenção, indicando que somente o irmão ganhava atenção e "regalias". Foi diagnosticado com transtorno de ansiedade generalizada (American Psychiatric Association, 2014).

A psicoterapeuta era psicóloga, tinha dois anos de experiência clínica no início do atendimento. Cursou mestrado em Psicologia Clínica.

\subsection{INSTRUMENTOS}

Child Psychotherapy Q-Set (CPQ) (Schneider, \& Jones, 2006, 2012): procedimento baseado na metodologia $\mathrm{Q}$, que tem como objetivo proporcionar uma avaliação-padrão para descrição e análise do processo psicoterápico. Foi delineado para ser usado com crianças de 3 a 13 anos de idade. É constituído por cem itens, sendo que cada um apresenta um enunciado que retrata uma característica importante do processo psicoterapêutico. Os itens representam: (a) atitudes da criança (sentimentos, comportamentos ou experiência), (b) ações e atitudes do terapeuta, e (c) a natureza da interação da díade. A metodologia Q permite a caracterização sistemática de uma multiplicidade de fenômenos e de interaçóes entre paciente e terapeuta, que podem estar presentes numa sessão de psicoterapia. A elaboração dos itens que fazem parte do procedimento resultou de um trabalho de pesquisa, de uma extensa revisão de literatura sobre psicoterapia de crianças e de outros instrumentos existentes com a mesma finalidade (Schneider, \& Jones, 2006, 2012).

O manual de codificação que acompanha os cem itens do CPQ apresenta explicações claras, assim como exemplos de cada item. Permite, portanto, maior confiabilidade e reflete particularidades que podem ser reconhecidas utilizando videotapes das sessóes de psicoterapia de crianças. O CPQ foi traduzido e adaptado para o português brasileiro (Ramires, \& Schneider, 2016). A fidedignidade e validade do CPQ vêm sendo demonstradas por diferentes investigações. Sua validade discriminante foi demonstrada em alguns estudos (Goodman, Midgley, \& Schneider, 2015; Schneider et al., 2009), assim como a fidedignidade interavaliadores (Carvalho, et al., 2016; Gastaud, Carvalho, Goodman, \& Ramires, 2015; Goodman, \& Athey-Lloyd, 2011; Goodman, et 
al. , 2015; Ramires, Carvalho, Schmidt, Fiorini, \& Goodman, 2015; Schneider et al., 2010). Em todos eles, análises independentes de grupos de sessões de psicoterapia de crianças realizadas por avaliadores treinados atingem índices de concordância superiores a 0,70 (correlação intraclasse).

\subsection{PROCEDIMENTOS}

Os pais do menino solicitaram atendimento, tendo sido realizada uma avaliação para confirmar a necessidade e motivação para o tratamento. Confirmada a indicação para a psicoterapia, por ocasião do estabelecimento do contrato, a proposta do estudo foi explicada para os pais e para a criança. A terapeuta já havia sido previamente consultada, tendo concordado em participar do estudo. Os pais e a terapeuta assinaram o termo de consentimento livre e esclarecido. O protocolo de pesquisa foi aprovado por Comitê de Ética em Pesquisa credenciado. Todos os procedimentos éticos foram respeitados, priorizando-se o bem-estar dos participantes, que estavam cientes de que poderiam desistir de participar a qualquer momento, sem prejuízo da psicoterapia em andamento.

A psicoterapia realizada tinha enfoque psicanalítico, na perspectiva da teoria das relações objetais, e foi realizada em consultório particular, com frequência de uma sessão semanal com duração de 50 minutos. Periodicamente, com a mesma terapeuta, foram realizadas entrevistas de acompanhamento com os pais.

Todas as sessões do menino foram gravadas em vídeo, com o consentimento da terapeuta, do paciente e dos pais. O dispositivo de filmagem foi uma pequena câmera, de tamanho aproximado ao de uma pequena máquina fotográfica, posicionada no consultório, em local previamente comunicado à criança. No total, foram realizadas, filmadas e analisadas 58 sessões de psicoterapia.

Cada sessão da psicoterapia de Antônio foi codificada de acordo com o CPQ, por dois juízes, de forma independente, formando pares aleatórios e que se intercambiaram. A equipe de juízes foi composta por seis psicólogos, treinados para o uso do CPQ, com experiência clínica em psicoterapia psicanalítica. A concordância entre qualquer par de juízes foi de pelo menos 0,70 (alpha de Cronbach) e variou de 0,62 a $0,85(\mathrm{~m}=0,73$ e $\mathrm{DP}=0,06)$ nas 58 sessões do tratamento. Com as classificações feitas pelos pares de juízes, calculou-se a média de cada sessão para formar escores compostos utilizados em todas as análises subsequentes.

Os escores compostos foram utilizados para calcular a média e o desviopadrão dos itens mais e menos característicos do $\mathrm{CPQ}$, de forma a descrever o processo terapêutico globalmente. A correlação de Pearson foi empregada para 
analisar a variação dos itens ao longo do tempo, de forma a identificar mudanças na interação terapeuta-paciente, e mudanças na criança e na terapeuta durante $o$ processo. Essas análises foram realizadas utilizando o SPSS 23.0.

\section{RESULTADOS}

\subsection{DESCRIÇÃO DO CASO}

Inicialmente, apresenta-se uma síntese da psicoterapia, baseada no relato da terapeuta, no intuito de contextualizar o processo terapêutico do menino. No início do processo, Antônio mostrou-se uma criança tímida, retraída e silenciosa. Chegava e sentava-se na poltrona, esperando que a terapeuta iniciasse a sessão. Esfregava as mãos, respondia a tudo que lhe era perguntado, mas o fazia de forma rápida e bastante sucinta.

Nas primeiras sessões, após estímulo da terapeuta, Antônio explorou um pouco o consultório, e jogou alguns jogos como xadrez e jogo da vida. Porém, desde o início, mostrou preferência pelo jogo de futebol de botôes.

Ao longo do tempo, Antônio expressou com clareza a falta que sentia do pai, reclamando que este nunca tinha tempo disponível para ficar com ele. Também falou sobre como se incomodava, às vezes, com seu irmão, quando tinha de fazer algumas coisas em razão dele (por exemplo, fazer café para ele e para o irmão; esperar que o irmão terminasse as refeições para que ele pudesse terminar também; caso contrário, o irmão chorava e gritava).

Antônio também falou sobre como se sentia exigido pela mãe em relação à escola, à organização da casa, aos cuidados com o irmão. Relatou um episódio no qual a mãe o inscreveu num curso preparatório para ingresso numa escola militar e disse: "A minha mãe deseja mais do que eu que eu entre nessa escola". Para a terapeuta, parecia que tanto o pai quanto a mãe tinham dificuldade para perceber as necessidades e os desejos do filho.

No decorrer do tratamento, Antônio passou a ficar mais competitivo e ganhar era uma necessidade. Passou a escolher, quase sempre, o jogo de botóes, no qual ele sabia ser muito bom. As tentativas da terapeuta de explorar o significado desse jogo e de sua necessidade de ganhar não surtiam efeito.

Por volta da vigésima sessão, o menino tornou-se mais retraído e contido nas sessões. O tema futebol passou a ser constante, e o jogo de botóes era sempre a atividade escolhida pelo paciente. Antônio evitava falar sobre seus sentimentos 
e pensamentos. Segundo a terapeuta, ele adotou um distanciamento maior na relação terapêutica e se defendia contra materiais que sentia como perturbadores. O sentimento da terapeuta, em diversos momentos, foi de exclusão, como se Antônio estivesse se refugiando dentro dele mesmo e não quisesse manter contato.

A ansiedade de Antônio foi aumentando com o passar do tempo, e alguns sintomas obsessivos começaram a ficar evidentes. O paciente fechava os olhos para não ver uma jogada, torcia para que a terapeuta errasse no jogo, batia na mesa para dar sorte, punia-se quando errava uma jogada. Quando a terapeuta tentava abordar isso, Antônio negava ou ignorava suas intervençōes, desejando apenas jogar. $\mathrm{O}$ paciente parecia estar buscando um maior controle da expressão de suas emoções e evitar se deparar com elas. Segundo relato da terapeuta, o menino parecia querer se proteger das emoções negativas e da angústia que elas provocavam. A análise de sua resistência e defesas não surtiram efeito.

Os pais foram acompanhados pela mesma terapeuta em entrevistas que visavam a fortalecer a aliança terapêutica, acolher suas preocupações em relação ao filho e trabalhar suas dúvidas sobre como lidar com ele. Foram realizadas nove entrevistas ao longo da psicoterapia. O pai, desde o início, mostrou-se resistente e contrariado, explicitando que não acreditava que pudesse ajudar e que não gostaria de estar ali. O casal brigava muito, tendo sido relatado que isso acontecia na presença dos filhos.

A mãe relatou ser uma pessoa perfeccionista e queixou-se muito da falta de ajuda do pai. O pai disse estar sempre muito cansado quando chegava em casa, e queixou-se do perfeccionismo e exigências da mãe. Não costumava fazer atividades com os filhos.

De acordo com a mãe, ao longo da psicoterapia, Antônio tornou-se mais calmo, embora ainda se irritasse quando contrariado e pudesse ser agressivo em alguns momentos. Passou a conversar mais com ela, organizou-se mais em relação às suas tarefas escolares e tornou-se mais maduro e autônomo. $\mathrm{O}$ pai não percebeu nenhuma mudança. Após 22 meses de psicoterapia, em razão de uma mudança de cidade da terapeuta, ela encaminhou Antônio para outra profissional, para que pudesse continuar sua psicoterapia. No entanto, pelas informações obtidas com a profissional, a família e o menino não buscaram esse atendimento.

\section{O processo terapêutico de acordo com o Child Psychotherapy Q-set}




\section{Tabela 1 - Quinze itens do CPQ mais e menos característicos ao longo do processo terapêutico} de Antônio

\section{Itens mais característicos}

\begin{tabular}{cllll}
\hline Item & \multicolumn{1}{c}{ Definição } & Média & DP & Variação \\
\hline 6 & T é sensível aos sentimentos da criança. & 7,93 & 0,77 & $5,5-9$ \\
23 & A sessão terapêutica tem um tema ou foco específico. & 7,87 &, 73 & $4,5-9$ \\
31 & T solicita mais informação ou elaboração. & 7,73 & 1,13 & $2-9$ \\
29 & A qualidade do brincar da C é fluida, absorta (vs. fragmentada, esporádica). & 7,72 & 0,93 & $5-9$ \\
88 & O material da sessão é significativo e relevante em relação aos conflitos da C. & 7,59 & 0,85 & $4-9$ \\
64 & C inclui o terapeuta no jogo. & 7,55 & 1,01 & $4,5-9$ \\
77 & A interação do T com a C é sensível a seu nível de desenvolvimento. & 7,55 & 0,95 & $4,5-9$ \\
39 & C é competitiva, rivaliza com o T. & 7,4 & 1,51 & $3-9$ \\
50 & T chama a atenção para os sentimentos considerados inaceitáveis por C (ex.: & 7,33 & 1,10 & $4-9$ \\
& raiva, inveja ou excitação). & 7,28 & 0,95 & $4,5-9$ \\
86 & T é confiante, seguro de si (vs. incerto ou inseguro). & 7,23 & 0,98 & $4-9$ \\
65 & T clarifica, reafirma ou reformula comunicações da criança. & 7,19 & 1,31 & $2-9$ \\
97 & T enfatiza a verbalização dos estados internos e afetos. & 7,09 & 1,29 & $3-9$ \\
28 & T percebe acuradamente o processo terapêutico. & 7,05 & 2,09 & $1-9$ \\
58 & C parece relutante em examinar pensamentos, reações ou motivações & 6,77 & 1,21 & $3-9$ \\
\hline & relacionadas aos problemas. & A autoimagem da C é um tema. &
\end{tabular}

\section{Itens menos característicos}

\begin{tabular}{cllll}
\hline Item & \multicolumn{1}{c}{ Definição } & Média & DP & Variação \\
\hline 9 & T não é responsivo (vs. afetivamente engajado). & 1,55 & 0,69 & $1-4$ \\
18 & T é crítico e transmite falta de aceitação. & 1,94 & 0,57 & $1-3,5$ \\
17 & $\begin{array}{l}\text { T ativamente exerce controle sobre a interação (ex.: estruturando, } \\
\text { introduzindo novos tópicos). }\end{array}$ & 1,97 & 0,97 & $1-6$ \\
32 & C alcança uma nova compreensão ou insight. & 2,34 & 1,16 & $1-6$ \\
53 & C expressa consciência das próprias dificuldades internas. & 2,76 & 1,62 & $1-7$ \\
5 & C tem dificuldade para compreender os comentários do T. & 3,06 & 1,13 & $1-6,5$ \\
26 & Cé socialmente não sintonizada ou inapropriada. & 3,08 & 1,11 & $1-7,5$ \\
71 & C se engaja no jogo de faz de conta. & 3,1 & 1,46 & $1-9$ \\
89 & T age para fortalecer as defesas existentes. & 3,24 & 1,77 & $1-8,5$ \\
41 & C não se sente compreendida pelo T. & 3,29 & 0,81 & $1,5-5$ \\
12 & T modela emoções não ditas ou não elaboradas. & 3,3 & 1,68 & $1-8,5$ \\
20 & C é provocadora; desafia o terapeuta ou as regras e limites da sessão. & 3,34 & 0,73 & $2-5$ \\
49 & C transmite ou expressa sentimentos confusos ou conflituosos sobre o T. & 3,34 & 0,70 & $2-5$ \\
37 & T se comporta de uma maneira didática. & 3,39 & 1,22 & $1-6,5$ \\
83 & C é exigente. & 3,59 & 0,89 & $2-6$ \\
\hline
\end{tabular}

$\mathrm{T}=$ Terapeuta, $\mathrm{C}=$ Criança.

Fonte: elaborado pelas autoras. 
A tabela 1 sintetiza as principais características do processo terapêutico de Antônio, de acordo com o CPQ, com base na média dos itens ao longo das 58 sessões realizadas e analisadas. $\mathrm{O}$ menino mostrou-se hesitante para abordar suas dificuldades de relacionamento e para pensar sobre sentimentos e emoçôes relacionados a seus problemas. Não tinha consciência de suas dificuldades nem alcançou insights sobre elas. Mostrou-se competitivo, embora se esforçasse para controlar seus impulsos. Ao longo do tratamento, tornou-se mais resistente às intervenções da terapeuta e mais distante em sua relação com ela. Por outro lado, incluía a terapeuta no jogo, o qual ocorria de maneira fluida e absorta. Era uma criança cordata, que respeitava os limites, que não brincava de maneira imaginativa e simbólica durante sua psicoterapia (ver itens 5, 20, 26, 29, 32, 35, $39,53,58,64,71$ e 83$)$.

Em relação à terapeuta, pode-se observar uma postura empática e sensível aos sentimentos da criança e a seu nível de desenvolvimento. Evidenciou uma atitude exploratória e colocou ênfase nos estados afetivos, chamando a atenção para sentimentos considerados inaceitáveis pela criança. Mostrou segurança em sua postura e atitudes e buscou enfatizar a verbalização dos estados internos e afetos do paciente. Aliado a isso, a terapeuta percebia acuradamente o processo terapêutico e demonstrava uma aceitação não crítica do paciente. Essas características podem ser observadas na tabela 1 , com base nos itens $6,9,12,17$, $18,31,28,37,50,65,77,86,89$ e 97.

A respeito da interação terapeuta-paciente, percebe-se que as sessões tiveram geralmente um tema ou foco específico, e seu material era significativo e relevante em relação aos conflitos da criança, mesmo que isso não fosse trabalhado. Antônio transmitia a sensação de sentir-se compreendido pela terapeuta (ver itens 23, 41, 49 e 88).

Posteriormente, analisou-se, por meio da correlação de Pearson, a variação dos itens do CPQ relacionados à criança, à terapeuta e à interação terapeutapaciente, ao longo das 58 sessóes realizadas. A tabela 2 apresenta esses resultados. 
Tabela 2 - Variação dos itens do CPQ ao longo do tempo

\begin{tabular}{ll}
\hline \multicolumn{1}{c}{ Descrição dos Itens do CPQ } & \multicolumn{1}{c}{ C. de Pearson } \\
\hline \multicolumn{1}{c}{ Itens da criança } &, $468^{* *}$ \\
\hline $\begin{array}{l}\text { direção T (vs. expressa aprovação ou admiração). } \\
8 \text { - C é curiosa. }\end{array}$ &,$- 444^{* *}$ \\
10 - C procura maior intimidade com T. &,$- 403^{* *}$ \\
13 - C está animada ou excitada. &,$- 627^{* *}$ \\
19 - C pede conselhos ou informações. &,$- 360^{* *}$ \\
26 - C é socialmente não sintonizada ou inapropriada. &, $435^{* *}$ \\
29 - A qualidade do brincar da C é fluida, absorta (vs. fragmentada, &,$- 432^{* *}$ \\
esporádica). &, $495^{* *}$ \\
40 - C se comunica sem afeto. &, $429^{* *}$ \\
42 - C ignora ou rejeita os comentários e observações do T. &,$- 511^{* *}$ \\
54 - C é clara e organizada na sua expressão verbal. &, $372^{* *}$ \\
56 - C está distante dos seus sentimentos. &, $381^{* *}$ \\
70 - C se esforça para controlar sentimentos ou impulsos. &,$- 461^{* *}$ \\
72 - C é ativa. &,$- 462^{* *}$ \\
78 - C é complacente. &, $621^{* *}$ \\
94 - C se sente triste ou deprimida (vs. animada e alegre). & \\
\hline
\end{tabular}

\section{Itens do terapeuta}

2 - T comenta sobre o comportamento não verbal da C (ex.: postura corporal, gestos).

, $273^{*}$

6 - T é sensível aos sentimentos da C.

$-, 373^{* *}$

9 - T não é responsivo (vs. afetivamente engajado).

17 - T ativamente exerce controle sobre a interação (ex.: estruturando, introduzindo novos tópicos).

36 - T assinala o uso de defesas por parte da C.

, $372^{* *}$

48 - T coloca limites.

$-, 276^{*}$

65 - T clarifica, reafirma, ou reformula comunicações da C.

, $312^{*}$

77 - A interação do T com a C é sensível ao seu nível de desenvolvimento.

79 - T comenta as mudanças no humor ou nas emoções da C.

86 - T é confiante, seguro de si (vs. incerto ou inseguro).

97 - T enfatiza a verbalização dos estados internos e afetos.

, $340^{* *}$

\section{Itens da interação}

38 - T e C demonstram um vocabulário ou compreensão compartilhada quando se referem a eventos ou sentimentos.

49 - C transmite ou expressa sentimentos confusos ou conflituosos sobre T.

64 - $C$ inclui T no jogo.

69 - A situação de vida atual ou recente da C é enfatizada. 
74 - O humor é utilizado.

$-, 275^{*}$

90 - Os sonhos ou fantasias da C são discutidos.

$-, 371^{* *}$

$\mathrm{T}=$ terapeuta; $\mathrm{C}=$ criança. ${ }^{*} \mathrm{~A}$ correlação é significativa no nível 0,05 ( 2 extremidades). ${ }^{* *} \mathrm{~A}$ correlação é significativa no nível 0,01 (2 extremidades).

Fonte: elaborado pelas autoras.

Com base nessa análise, é possível observar que, com o passar do tempo, Antônio tornou-se mais triste e deprimido, menos cordato e mais hostil em relação à terapeuta, rejeitando suas intervenções. Houve um afastamento no relacionamento com ela, o jogo perdeu fluidez, e a expressão verbal do paciente tornou-se menos clara. Antônio passou a demonstrar maior afastamento de seus sentimentos (itens $1,8,10,13,19,26,29,40,42,54,56,70,72,78$ e 94).

Paralelamente, a terapeuta tornou-se menos responsiva e sensível aos sentimentos do paciente. Intensificou o assinalamento de suas defesas e passou a reformular mais suas comunicações. Exerceu maior controle sobre a interação e tornou-se mais incerta ou insegura (ver tabela 2, itens 2, 6, 9, 17, 36, 48, 65, 77, 79, 86 e 97).

Sobre a interação, os resultados mostraram que o menino, no decorrer do tratamento, expressou mais sentimentos conflituosos sobre a terapeuta e deixou de incluí-la no jogo. Houve maior afastamento na díade terapeuta-paciente, menos humor, e a discussão de acontecimentos sobre a vida da criança ou sobre seus sonhos e fantasias também experimentou um declínio (ver tabela 2, itens $38,49,64,69,74$ e 90$)$.

\section{DISCUSSÃO}

O processo da psicoterapia de Antônio testemunha a complexidade do tratamento de crianças com transtorno de ansiedade, frequentemente acompanhado por outras desordens (Bernstein, \& Shaw, 1997; Fonagy et al., 2005). Embora algumas melhoras tenham sido reportadas pela mãe do menino, o processo terapêutico retratado pelo CPQ revelou uma evolução marcada por gradativo afastamento na relação terapeutapaciente, resistência crescente por parte do menino e falta de abertura para análise das suas dificuldades, conflitos e mecanismos de defesa. Ao mesmo tempo, da parte da terapeuta, também foi possível observar mudanças nas características da sua conexão com o paciente, tornando-se menos responsiva, menos sensível a seus estados emocionais e menos flexível e segura.

Os poucos estudos disponíveis sobre o emprego da psicoterapia psicodinâmica com crianças que apresentavam transtornos internalizantes documentaram sua 
utilidade, embora seus autores tenham assinalado algumas reservas devido a limitações metodológicas (Bernstein, \& Shaw, 1997; Target, \& Fonagy, 1994). Trabalhar os medos e ansiedades subjacentes foi considerado um componente importante desses tratamentos assim como focalizar temas de separação, autonomia, autoestima e comportamentos apropriados para a idade (Bernstein, \& Shaw, 1997). Segundo os autores, os pais precisam ser envolvidos no tratamento, de forma a compreender a necessidade de reasseguramento da criança e encorajá-la a ser mais independente. Os pais podem também necessitar resolver suas próprias questóes sobre separação e outras fontes de ansiedade, porque eles podem exacerbar medos da criança ou comunicar-se com ela de maneira ambivalente sobre sua segurança e autonomia.

Essa abordagem parece não ter sido empregada de modo suficiente na psicoterapia de Antônio. O menino vivia numa família que apresentava algumas características disfuncionais, tais como conflitos entre o casal, dificuldades resultantes da sobrecarga de lidar com um filho autista, distanciamento do pai e sobrecarga da mãe. Esses fatores podem ter contribuído para as melhoras modestas observadas durante o processo e para algumas características da interação terapeuta-paciente observadas.

Contudo, além dos fatores externos ao setting clínico, é importante que se considere também os fatores da relação terapêutica bem como do paciente e da terapeuta. Os resultados do CPQ evidenciaram que houve um incremento da transferência negativa ao longo dos 22 meses da psicoterapia. Antônio tornou-se mais hostil e passou a rejeitar, de maneira crescente, as intervençôes da terapeuta. Seu jogo perdeu fluidez, e o afastamento de seus sentimentos e emoções se intensificou.

Uma hipótese possível para explicar tais resultados diz respeito à repetição, na psicoterapia, da vivência do menino com sua família. Antônio era uma criança que não se sentia suficientemente investido afetivamente pelos pais. Os conflitos do casal, o luto pelo filho mais novo diagnosticado com autismo, o isolamento do pai na família e as características rígidas e exigentes da mãe contribuíam para uma vivência de abandono e desamparo, insegurança, baixa autoestima, que parecem não ter sido suficientemente compreendidas, analisadas e interpretadas durante o processo terapêutico.

Conteúdos importantes não foram interpretados e trabalhados, o que pode ter sido experimentado pelo menino como uma repetição da vivência de desinvestimento e abandono possivelmente sentidos em sua família. Conforme o CPQ, ao longo da psicoterapia, Antônio tornou-se, de maneira significativa, mais triste e deprimido, com mais dificuldade de expressar seus sentimentos e emoçōes, mais hostil, e seu jogo perdeu espontaneidade. Além disso, alguns traços obsessivos passaram a ser observados.

Fonagy et al. (2005) assinalaram que a psicoterapia deve oferecer oportunidades para a criança articular pensamentos, sentimentos, lembranças e desejos, bem como para fazer conexões entre seus sentimentos e conflitos inconscientes. A capacidade de 
mentalização seria o alicerce para que essas articulações e conexões pudessem ocorrer. Trata-se de um constructo elaborado no contexto da teoria do apego, como um processo desenvolvimental, por meio do qual a criança desenvolve a capacidade de interpretar o próprio comportamento e o dos outros, considerando os estados mentais subjacentes, processo mediado pelos cuidadores primários (Fonagy, Gergely, Jurist, \& Target, 2002). A promoção da capacidade de mentalização foi descrita como um fator comum, inerente a todos os modelos de tratamento efetivos (Allen, Fonagy, \& Bateman, 2008; Goodman, Midgley, \& Schneider, 2015).

Levanta-se a hipótese de que Antônio pudesse apresentar limitaçóes em sua capacidade de mentalização quando iniciou sua psicoterapia. Esse processo necessitaria, então, ser promovido para que a análise das dificuldades e conflitos do paciente fossem possíveis. Contudo a falta de mentalização do paciente pode contaminar o terapeuta, tornando-o refém de uma intrincada rede de signos não compreendidos. Isso pode ter acontecido no processo terapêutico de Antônio, contribuindo para explicar as mudanças em sua postura e intervenções descritas na tabela 2. Se, em média, durante os 22 meses de psicoterapia, a terapeuta caracterizou-se pela postura empática e sensível, tendo evidenciado uma atitude exploratória e enfatizando os estados afetivos do paciente, chamando atenção para seus sentimentos, intervenções características da abordagem baseada na mentalização (Allen, Fonagy, \& Bateman, 2008; Goodman et al., 2015), com o passar do tempo, tal postura e intervenções mostraram um declínio.

Birmaher e Brent (2007) discutiram fatores que devem ser considerados com relação aos pacientes que não estão respondendo ao tratamento. Entre esses fatores, os autores destacaram diagnóstico errado, comorbidades não reconhecidas ou não tratadas, modalidade psicoterápica inapropriada, intensidade ou duração inapropriada do tratamento, falta de adesão ao tratamento, exposição a crônicos ou severos eventos contextuais (abusos, conflitos familiares) e nível de habilidades inadequado ou falta de ajuste do terapeuta. Nesse sentido, além dos fatores já discutidos para explicar o processo terapêutico de Antônio, da forma como foi detectado pelo CPQ, também devem ser considerados a contratransferência eventualmente não analisada da terapeuta e o fato de estar no início da sua carreira profissional, nos primeiros anos de experiência clínica. Mesmo buscando recursos para o aprimoramento de sua atividade, como supervisão e formação teórica, além do tratamento pessoal, Antônio era um paciente que impunha importantes desafios. Adicionalmente, não deve ser desprezada a variável da filmagem das sessões. Embora esse fator não tenha sido objeto de questionamento pela dupla durante o processo terapêutico, pode ter gerado algum desconforto consciente ou inconscientemente, contribuindo para o desfecho observado. 


\section{CONSIDERAÇÕES FINAIS}

Embora a literatura venha sinalizando a efetividade da psicoterapia psicodinâmica para pacientes com transtornos internalizantes (Midgley, \& Kennedy, 2011; Palmer et al., 2013), o processo terapêutico analisado neste estudo caminhava em direção oposta até o momento em que foi interrompido. Possivelmente, tal evolução não se explica com base num único fator, sendo possível levantar a hipótese de que todas as alternativas discutidas acima contribuíram, mesmo que em diferentes dimensões, para explicar o andamento da psicoterapia.

Por outro lado, é importante reconhecer que o paciente e seus pais estabeleceram uma aliança com a terapeuta, que permitiu manter a psicoterapia por aproximadamente dois anos, apesar das dificuldades e características descritas acima. Considera-se que a evolução do processo observada não significa que a psicoterapia psicodinâmica não seja efetiva para crianças com desordens internalizantes. Ao contrário, ela alerta para fatores importantes que necessitam ser monitorados e contemplados, no sentido de buscar essa efetividade. Entre esses fatores, destacaram-se: condições e conflitos familiares, importância do estabelecimento da aliança terapêutica com ambos os pais, acurácia do diagnóstico e adequação das intervenções técnicas ao nível de desenvolvimento e das dificuldades da criança, diagnóstico de sua capacidade de mentalização e expertise da terapeuta, que inclui formação teórica, técnica e habilidade para compreensão e manejo da contratransferência.

A utilização de diferentes instrumentos e fontes de informação poderia contribuir para superar algumas das limitaçóes deste estudo. Por exemplo, entrevistar a terapeuta, os pais e a própria criança, escutando-os acerca da forma como experimentaram o processo terapêutico, poderia lançar luz sobre aspectos não contemplados por um procedimento como o CPQ. Futuras investigações poderão contemplar o estudo da psicoterapia de crianças com transtornos internalizantes com base em delineamentos experimentais e outros, explorando diferentes aspectos do processo como aliança terapêutica, transferência e contratransferência, estratégias técnicas adotadas, turning points, etc.

Finalmente, cabe assinalar que, se a pesquisa naturalística, tal como empregada nesta investigação, pode constituir um risco ou interferência para o processo terapêutico, por outro lado, ela é movida pelo compromisso ético e pela necessidade de contribuir para elucidar questôes em aberto acerca da efetividade das psicoterapias e dos seus mecanismos de mudança. Somente conhecendo e compreendendo, de fato, o que acontece e como acontece no setting clínico será possível estabelecer uma base de evidências consistente para a psicoterapia psicodinâmica. 


\section{REFERÊNCIAS}

Achenbach, T. M. (1991). Manual for the Child Behavior Checklist/4-18 e 1991 profile. Burlington: University of Vermont.

Allen, J. G., Fonagy, P., \& Bateman, A. W. (2008). Mentalizing in clinical practice. Arlington: American Psychiatric Publishing.

American Psychiatric Association [APA]. (2014). Manual diagnóstico e estatístico de transtornos mentais: DSM-5. (M. I. C. Nascimento et al. Trad.) (5a ed.). Porto Alegre: Artmed.

Bayer, J. K., Rapee, R. M., Hiscock, H., Ukoumunne, O. C., Mihalopoulos, C., \& Wake, M. (2011). Translational research to prevent internalizing problems early in childhood. Depression and Anxiety, 28(1), 50-57. Recuperado a partir de https://doi.org/10.1002/da.20743

Bernstein, G. A., \& Shaw, K. (1997). Practice parameters for the assessment and treatment of children and adolescents with anxiety disorders. Journal of the American Academy of Child Adolescent Psychiatry, 36(10), 69S-84S. Recuperado a partir de https://linkinghub.elsevier.com/retrieve/pii/S0890856709625958

Birmaher, B., \& Brent, D. (2007). Practice parameter for the assessment and treatment of children and adolescents with depressive disorders. Journal of the American Academy of Child e Adolescent Psychiatry, 46(11), 15031526. Recuperado a partir de https://linkinghub.elsevier.com/retrieve/pii/ S0890856709625958

Boyd, C. P., Kostanski, M., Gullone, E., Ollendick, T. H., \& Schek, D. T. L. (2000). Prevalence of anxiety and depression in Australian adolescents: comparison with worldwide data. The Journal of Genetic Psychology, 161(4), 479-492. Recuperado a partir de https://www.tandfonline.com/doi/ abs/10.1080/00221320009596726

Carvalho, C., Godinho, L. R., \& Ramires, V. R. R. (2016). O processo psicoterapêutico de uma criança: análise baseada no Child Psychotherapy Q-Set. Temas em Psicologia, 24(3), 1153-1167. Recuperado a partir de http://pepsic. bvsalud.org/pdf/tp/v24n3/v24n3a19.pdf

Deakin, E. K., Gastaud, M. B., \& Nunes, M. L. T. (2012). Child psychotherapy dropout: an empirical research review. Journal ofChild Psychotherapy, 38(2), 199209. Recuperado a partir de https://doi.org/10.1080/0075417X.2012.684489 
Deakin, E. K., \& Nunes, M. L. T. (2008). Investigação em psicoterapia com crianças: uma revisão. Revista de Psiquiatria do Rio Grande do Sul, 30 (1 Supl.). Recuperado a partir de https://doi.org/10.1590/S0101-81082008000200003

Edwards, D. J. A. (2007). Collaborative versus adversarial stances in scientific discourse: implications for the role of systematic case studies in the development of evidence-based practice in psychotherapy. Pragmatic Case Studies in Psychotherapy, 3(1), 6-34. Recuperado a partir de https://pcsp.libraries.rutgers. edu/index.php/pcsp/article/view/892/2260

Eizirik, C. L., \& Hauck, S. (2008). Psicanálise e psicoterapia de orientação psicanalítica. In A. V. Cordioli (Org.), Psicoterapias: abordagens atuais (pp. 151-166). (3a ed.). Porto Alegre: Artmed.

Flett, G. L., \& Hewitt, P. L. (2013). Disguised distress in children and adolescents "flying under the radar": why psychological problems are underestimated and how schools must respond. Canadian Journal of School Psychology, 28(1), 1227. Recuperado a partir de https://doi.org/10.1177/0829573512468845

Fonagy, P., Gergely, G., Jurist, E. L., \& Target, M. (2002). Affect regulation, mentalization, and the development of the self. New York: Other Press.

Fonagy, P., Target, M., Cottrell, D., Phillips, J., \& Kurtz, A. (2005). Anxiety Disorders. In P. Fonagy (Ed.), What works for whom?: a critical review of treatments for children and adolescents. (pp. 66-88). New York: Guilford Press.

Gastaud, M. B., Carvalho, C., Goodman, G., \& Ramires, V. R. (2015). Assessing levels of similarity to a "psychodynamic prototype" in psychodynamic psychotherapy with children: a case study approach (preliminary findings). Trends in Psychiatry in Psychotherapy, 37(3), 161-165. Recuperado a partir de https://doi.org/10.1590/2237-6089-2014-0059

Goldmann, S. (2012). Developmental epidemiology of depressive disorders. Child and adolescent psychiatric clinics of North America, 21(2), 217-235. Recuperado a partir de https://www.sciencedirect.com/science/article/abs/pii/ S1056499311001349?via\%3Dihub

Goodman, G., \& Athey-Lloyd, L. (2011). Interaction structures between a child and two therapists in the psychodynamic treatment of a child with Asperger's disorder. Journal of Child Psychotherapy, 37(3), 311-326. Recuperado a partir de https://www.tandfonline.com/doi/abs/10.1080/0075417X.2011.614749 
Goodman, G., Midgley, N., \& Schneider, C. (2015). Expert clinicians' prototypes of an ideal child treatment in psychodynamic and cognitive-behavioral therapy: is mentalization seen as a common process factor?. Psychotherapy Research, 26(5), 1-12. Recuperado a partir de https://www.tandfonline.com/doi/full/10 $.1080 / 10503307.2015 .1049672$

Göttken, T., White, L. O., Klein, A. M., \& Klitzing, K. V. (2014). Shortterm psychoanalytic child therapy for anxious children: a pilot study. Psychotherapy, 51(1), 148-158. Recuperado a partir de https:/doi.apa.org/ doiLanding? $\mathrm{doi}=10.1037 \% 2 \mathrm{Fa} 0036026$

Mash, E. J., \& Hunsley, J. (2010). Assessment of childhood disorders. In E. J. Mash, \& R. A. Barkley (Eds.). Assessment of childhood disorders. (pp. 3-51). New York: Ghilford.

Merry, S. N., Hetrick, S. E., Cox, G. R., Brudevold Iversen, T., Bir, J. J., \& McDowell, H. (2012). Psychological and educational interventions for preventing depression in children and adolescents. Evidence Based Child Health: a Cochrane Review Journal, 7(5), 1409-1685. Recuperado a partir de https://doi.org/10.1002/ebch.1867

Midgley, N., \& Kennedy, E. (2011). Psychodynamic psychotherapy for children and adolescents: a critical review of the evidence base. Journal of Child Psychotherapy, 37(3), 232-260. Recuperado a partir de 1https://www. tandfonline.com/doi/abs/10.1080/0075417X.2011.614738

Milrod, B., Shapiro, T., Gross, C., Silver, G., Preter, S., Libow, A., \& Leon, A. C. (2013). Does manualized psychodynamic psychotherapy have an impact on youth anxiety disorders?. American Journal of Psychotherapy, 67(4), 359-366. Retrieved from: http://www.ingentaconnect.com/content/afap/ ajp/2013/00000067/00000004/art00004

Muratori, F., Picchi, L., Bruni, G., Patarnello, M., \& Romagnoli, G. (2003). A two-year follow-up of psychodynamic psychotherapy for internalizing disorders in children. Journal of the American Academy of Child and Adolescent Psychiatry, 42(3), 331-339. Recuperado a partir de https://doi.org/10.1097/00004583200303000-00014

Palmer, R., Nascimento, L. N., \& Fonagy, P. (2013). The state of the evidence base for psychodynamic psychotherapy for children and adolescents. Child and Adolescent Psychiatric Clinics of North America, 22(2), 149-214. Recuperado a partir de https://doi.org/10.1016/j.chc.2012.12.001 
Ramires, V. R. R., Carvalho, C., Schmidt, F. M. D., Fiorini, G. P., \& Goodman, G. (2015). Interaction structures in the psychodynamic therapy of a boy diagnosed with Asperger's disorder: a single-case study. Research in Psychotherapy: Psychopathology, Process and Outcome, 18(2), 129-140. Recuperado a partir de https://doi.org/10.4081/ripppo.2015.195

Ramires, V. R. R., \& Schneider, C. (2016). Psicoterapia de crianças: desenvolvimento da versão em português do Child Psychotherapy Q-Set Psicologia: Teoria e Pesquisa, 32(3). Recuperado a partir de https://doi. org/10.1590/0102-3772e323218

Schmidt, F. M. D. (2016). Análise do processo psicoterapêutico de uma criança com transtorno de adaptação e uma terapeuta grávida. (Dissertação de Mestrado). Universidade do Vale do Rio dos Sinos, São Leopoldo.

Schneider, C., \& Jones, E. E. (2006). Child Psychotherapy Q-Set: coding manual. Berkeley: University of California.

Schneider, C., \& Jones, E. E. (2012). Appendix IB: Child Psychotherapy Q-Set. Coding Manual. In R. A. Levy, J. S. Ablon, \& H. Kächele (Eds), Psychodynamic psychotherapy research: evidence-based practice and practice-based evidence. (pp. 611-626). New York: Human Press.

Schneider, C., Midgley, N., \& Duncan, A. (2010). A "motion portrait" of a psychodynamic treatment of an 11-year-old girl: exploring interrelations of psychotherapy process and outcome using the Child Psychotherapy Q-Set. Journal of Infant, Child and Adolescent Psychotherapy, 9(2-3), 94-107. Recuperado a partir de https://www.tandfonline.com/doi/abs/10.1080/15289 168.2010.510979

Schneider, C., Pruetzel-Thomas, A., \& Midgley, N. (2009). Discovering new ways of seeing and speaking about psychotherapy process: The Child Psychotherapy Q-Set. In N. Midgley, J. Anderson, E. Grainger, T. VuckovicNesic, \& C. Urwin (Eds.), Child psychotherapy and research: new approaches, emerging findings. (pp. 72-84). New York: Routledge.

Target, M., \& Fonagy, P. (1994). Efficacy of psychoanalysis for children with emotional disorders. Journal of the American Academy of Child Adolescent Psychiatry, 33(3), 361-371. Recuperado a partir de https://doi. org/10.1097/00004583-199403000-00010

Wehry, A. M., Beesdo-Baum, K., Hennelly, M. M., Connolly, S. D., \& Strawn, 
J. R. (2015). Assessment and treatment of anxiety disorders in children and adolescents. Current Psychiatry Reports, 17(7), 52. Recuperado a partir de https://doi.org/10.1007/s11920-015-0591-z

Wilkinson, P. (2009). Conceptualization about internalizing problems in children and adolescents. Ciência \& Saúde Coletiva, 14(2), 373-381. Recuperado a partir de https://doi.org/10.1590/S1413-81232009000200007 